Um festzustellen, wie viel Substanz aus dem schon einmal der Chlorkalzium-Destillation unterworfenen Melilotus-Extrakt durch erneuerte Destillation äbergehen würde, habe ich zahlreiche Versuche angestellt. $\mathrm{Zu}$ diesem Zwecke wurden zu der erstarrten Masse $200 \mathrm{~cm}$ destilliertes Wasser gegeben und wieder bis zum Erstarren destilliert. Die Mengen der bei dieser Gelegenheit übergegangenen Stoffe ergeben nach gravimetrischen wie nach titrimetrischen Bestimmungen $0,025-0,03^{0}$, und wie an den bei den gravimetrischen Bestimmungen erhaltenen Substanzen zu sehen war, enthielten diese schon sehr viel Verunreinigungen.

Nun will ich meine Methode der Kumarinbestimmungen auf die verschiedenen Melilotus-Arten and Individuen anwenden und dann das Verhalten des Kumarins während der Entwicklung der Pflanze und auch eventuell das Werden und Vergehen anderer Kumarsäure-Verbindungen in den verschiedenen Arten oder während verschiedener Entwicklungsperioden studieren.

\title{
Jahresbericht der internationalen Atomgewichtskommission für 1913.
}

Seit dem Jahresbericht für 1912 ist eine AnzahI von wichtigen Abhandlungen über die Atomgewichte erschienen. Auch liegen einige frühere Arbeiten vor, welche zu spät anlangten, um damals erwähnt zu werden. Folgendermaßen können diese Arbeiten zusammengefasst werden:

Stickstoff. Wourtzel ${ }^{1}$ ) hat das Verhältnis zwischen Stickstoff und Sauerstoff durch die Oxydation von Stickoxyd zu Stickstoffperoxyd neu bestimmt. Fünf übereinstimmende Messungen ergeben im Mittel: $\mathrm{N}=14,0068$.

Kalium und Chlor. Staehler und Meyer ${ }^{z}$ ) haben sorgfältige Analysen von Kaliumchlorat gemacht, wobei sie besondere Vorsichtsmaßregeln gegen die Verunreinigung durch das Chlorid anwendeten. Ihre Schlussreihen ergeben im Mittel $\mathrm{K} \mathrm{Cl}=74,5551$, woraus $\mathrm{K}=39,097$ and $\mathrm{Cl}=35,458$ folgt. Bezüglich der Diskussion ihrer Ergebnisse ist auch $\mathrm{Guye} \mathrm{e}^{3}$ ) nachzusehen, welcher schliesst, dass die oben erwähnte

1) Comptes rendus 154, 115 (1912).

2) Zeitschrift f. anorgan. Chemie 71, 378 (1912); diese Zeitschrift 51, 403.

3) Journ. Chim. Phys. 10, 145 (1912). 
Verunreinigung, wenn auch vielleicht nicht vollständig, so doch jedenfalls genügend beseitigt worden ist, um sie praktisch vernachlässigen zu können.

Fluor. Mc Adam and $\mathrm{Smith}{ }^{1}$ ) haben zwei vorläufige Bestimmungen des Atomgewichts des Fluors veröffentlicht. Natriumfluorid wurde in das Chlorid verwandelt durch Erhitzen in trockenem, gasförmigem Chlorwasserstoff, und aus dem Verbältnis zwischen den Gewichten wurde das Atomgewicht berechnet. Die beiden gefundenen Werte sind $\mathrm{F}=19,0176$ und 19,0133 .

Phosphor. Aus Analysen von Phosphortribromid haben Baxter, Moore und Boylston ${ }^{2}$ ) gefunden, dass. im Mittel von drei Reihen $\mathrm{P}=31,027$ folgt, wenn $\mathrm{Ag}=107,88$ ist. Dies stimmt recht gut mit dem früheren Ergebnis von Baxter und Jones am Silberphospha überein. Weitere Untersuchungen über Phosphortrichlorid sind in Aussicht gestellt.

Quecksilber. Easley und $\mathrm{Braun}{ }^{3}$ ) haben durch die Analyse von Merkuribromid $\mathrm{Hg}=200,64$ gefunden. Dies bestätigt die frühere Bestimmung von Easloy an dem Chlorid.

Selen. Kuzina und $\mathrm{Krehlik}$ ) haben das Atomgewicht von Selen durch Reduktion von Selendioxyd mit Schwefeldioxyd neu bestimmt. Der Mittelwert von zehn Bestimmungen ist $\mathrm{Se}=79,26$.

Tellur. Harcourt und $\mathrm{Bakker}{ }^{5}$ ) haben die Untersuchungen von Flint in Zweifel gezogen, welcher den Anspruch erhoben hatte, das bisherige Element Tellur in zwei Fraktionen von verschiedenem Atomgewicht gespalten zu haben. Sie wiederholten seine Methode der Fraktionierung und fanden an der vierten Fraktion $\mathrm{Te}=127,54$. Dieses stimmt mit der Zahl überein, welche von Bakker und Bennett 1907 gefunden worden ist. Ähnliche Fraktionierungen sind auch von Pellini ${ }^{6}$ ) ausgeführt worden, welcher gleichfalls keinerlei Andeutung eines Tellurs von niedrigem Atomgewicht gefundon hat.

Radium. Hönigs chmid ${ }^{7}$ ) hat durch sorgfältige Analysen von verhältnismäß̊ig grossen Mengen Radiumchlorid Ra $=225,95$ gefunden.

1) Journal of the American chemical Society 34, 592 (1912).

2) Proc. Amer. Acad. 47, 585 (19.2); Journal of the American chemical Society 34, 259 (1912); diese Zeitschrift 51, 727.

3) Journal of the American chemical Society 34, 137 (1912).

4) Abh. der Kgl. Franz Josephs Akademie 19, Nr. 13 (1910). Mitgeteilt von Prof. B. Brauner.

5) Journal of the chemical Society 99, 1311 (1912).

6) Atti Acc. Lincei 21, 2.8 (1912).

7) Monatsh. f. Chemie 33, 253 (1912); diese Zeitschrift 51, 598. 
Andererseits haben Gray und Ramsay) unter Verwendung sehr kleiner Mengen von Material und durch Umwandlung des Bromids in das Chlorid $\mathrm{Ra}=226,36$ übereinstimmend mit frühern Bestimmungen von Frau Curie und Thorpe gefunden. Bevor dieser Unterschied zwischen Hönigschmid's niedrigem Werte und dem höheren erklärt ist, erscheint es nicht zweckmäßig, die in der Tabelle angegebene Zahl zu ändern.

Tantal. Die Bestimmungen dieses Atomgewichts von $\mathrm{Chapin}$ und und $\mathrm{Smith}^{2}$ ) sind durch die Hydrolyse von Tantalpentachromid gemacht worden. Der Mittelwert von acht Bestimmungen ergab $\mathrm{Ta}=181,80$, welche Zahl etwas böher ist als die von Balke aus ähnlichen Analysen des Pentachlorids gefundene.

Iridium. Hoyermann $\left.{ }^{3}\right)$ fand durch fünf Reduktionen von $\left(\mathrm{NH}_{4}\right)_{2}$ $\operatorname{Ir~} \mathrm{Cl}_{6}$ in Wasserstoff $\mathrm{Ir}=192,613$.

Holmium. Sechs Bestimmungen des Atomgewichts von Holmium

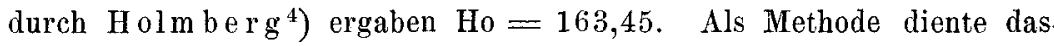
wohlbekannte Sulfatverfahren.

Ebenso liegen annähernde Bestimmungen der Atomgewichte von Blei, Zink und Kupfer durch $\mathrm{Pecheux}{ }^{5}$ ) und von Kalzium durch Oechsner de Coninck ${ }^{6}$ ) vor. Die erhaltenen Zahlen sind nicht entscheidend genug, um ihre Aufnahme in die Tabelle zu rechtfertigen, da die angewendeten Methoden keine grosse Genauigkeit verbürgen.

In der Tabelle für 1913 wird demgemäß nur eine Änderung vorgeschlagen, nämlich die Einfügung von Holmium, für welches bisher keine zuverlässige Atomgewichtsbestimmung vorlag. Zwei oder drei andere Änderungen von geringer Bedeutung könnten gemacht werden, doch scheint es nicht wünschenswert, solche Änderungen allzu häufig vorzunehmen.

\section{Gezeichnet}

Clarke, Thorpe, Ostwald, Urbain.

1) Proc. Roy. Soc. 86 A, 270 (1912).

2) Journal of the American chemical Society 33, 1497 (1911).

3) Sitzungsber. d. phys.-med. Soz. Erlangen 42, 278.

4) Zeitschrift f. anorgan. Chemie 71, 226 (1911).

5) Comptes rendus 154, 1419 (1912).

6) Comptes rendus 153, 1479 (1911); diese Zeitschrift 51, 600. 
194 Jahresbericht der internationalen Atomgewichtskommission für 1913.

Internationale Atomgewichte 1913.

\begin{tabular}{|c|c|c|c|c|c|c|c|c|c|c|c|}
\hline $\mathrm{Ag}$ & Silber & - & · & . & 107,88 & $N$ & Stickstoff & . & & & 14,01 \\
\hline $\mathrm{Al}$ & Aluminium & & - & . & 27,1 & $\mathrm{Na}$ & Natrium & & & & 23,00 \\
\hline $\mathrm{Ar}$ & Argon . & - & • & . & 39,88 & $\mathrm{Nb}$ & Niobium & . & & & 93,5 \\
\hline As & Arsen & . & . & . & 74,96 & $\mathrm{Nd}$ & Neudym. & . & & & 144,3 \\
\hline $\mathrm{Au}$ & Gold & . & . & . & 197,2 & $\mathrm{Ne}$ & Neon & . & & & 20.2 \\
\hline B & Bor . & . & . & . & 11,0 & $\mathrm{Ni}$ & Nickel . & . & & & 58,68 \\
\hline $\mathrm{Ba}$ & Baryum & . & • & . & 137,37 & $\mathrm{Nt}$ & Niton & $\cdot$ & & & 222,4 \\
\hline $\mathrm{Be}$ & Beryllium & . & . & . & 9,1 & 0 & Sauerstoff & . & & & 16,00 \\
\hline $\mathrm{Bi}$ & Wismut & 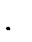 & . & . & 208,0 & $\mathrm{Os}$ & Osnium & . & & . & 190,9 \\
\hline $\mathrm{Br}$ & Brom & . & . & . & 79,92 & $\mathrm{P}$ & Phosphor & . & & & 31,04 \\
\hline $\mathrm{C}$ & Kohlenstoff & & . & . & 12,00 & $\mathrm{~Pb}$ & Blei . . & . & & & . 207,10 \\
\hline $\mathrm{Ca}$ & Kalzium & . & . & . & 40,07 & $\mathrm{Pd}$ & Palladium & . & & & 106,7 \\
\hline $\mathrm{Cd}$ & Kadmium & . & . & . & 112,40 & $\operatorname{Pr}$ & Praseodym & . & & . & 140,6 \\
\hline $\mathrm{Ce}$ & Cerium & . & . & . & 140,25 & Pt & Platin . & . & & - & 195,2 \\
\hline $\mathrm{Cl}$ & Chlor & - & . & . & 35,46 & Ra & Radium & . & & 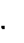 & 226,4 \\
\hline Co & Kobalt . & . & . & . & 58,97 & $\mathrm{Rb}$ & Rubidium & . & & 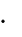 & 85,45 \\
\hline $\mathrm{Cr}$ & Chrom. & . & . & . & 52,0 & Rh & Rhodium & . & & . & 102,9 \\
\hline $\mathrm{Cs}$ & Cäsium . & . & . & . & 132,81 & $\mathrm{Ru}$ & Ruthenium & & & 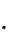 & - 101,7 \\
\hline $\mathrm{Cu}$ & Kupfer . & . & . & . & 63,57 & S & Schwefel & . & & & 32,07 \\
\hline Dy & Dysprosium & & . & . & 162,5 & $S a$ & Samarium & . & • & & 150,4 \\
\hline $\mathrm{Er}$ & Erbium & . & . & . & 167,7 & $\mathrm{Sb}$ & Antimon & . & - & & 120,2 \\
\hline $\mathrm{Eu}$ & Europium & . & . & . & 152,0 & $\mathrm{Sc}$ & Skandium & . & • & & 44,1 \\
\hline $\mathbf{F}$ & Fluor & . & . & . & 19,0 & $\mathrm{Se}$ & Selen & . & $\cdot$ & & 79,2 \\
\hline $\mathrm{Fe}$ & Eisen & . & . & . & 55,84 & $\mathrm{Si}$ & Silizium & . & - & & 28,3 \\
\hline $\mathrm{Ga}$ & Galliurn & . & . & . & 69.9 & $\mathrm{Sn}$ & Zinn. . & . & & & . 119,0 \\
\hline $\mathrm{Gd}$ & Gadolinium & & . & & 157,3 & $\mathrm{Sr}$ & Strontium & - & & . & 87,63 \\
\hline $\mathrm{Ge}$ & Germanium & & . & . & 72,5 & Ta & Tantal. & . & - & $\cdot$ & 181,5 \\
\hline $\mathrm{H}$ & Wasserstoff & & . & & 1,008 & $\mathrm{~Tb}$ & Terbium & . & . & • & . $\quad 159,2$ \\
\hline $\mathrm{He}$ & Helium . & & . & . & 3,99 & $\mathrm{Te}$ & Tellur. & . & • & • & 127,5 \\
\hline $\mathrm{Hg}$ & Quecksilber & & . & . & 200,6 & Th & Thorium & . & . & & 232.4 \\
\hline Ho & Holmium & 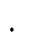 & . & . & 163,5 & $\mathrm{Ti}$ & Titan . & . & & & 48,1 \\
\hline In & Indium . & . & . & . & 114,8 & $\mathrm{Tl}$ & Thallinm & . & . & & 204,0 \\
\hline $\mathrm{Ir}$ & Iridium & 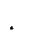 & . & . & 193,1 & 'I'u. & Thulium & : & . & & 168,5 \\
\hline $\mathbf{J}$ & Jod . . & 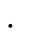 & . & . & 126,92 & $\mathrm{U}$ & Uran & . & . & & 238,5 \\
\hline $\mathrm{K}$ & Kalium & 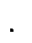 & . & . & 39,10 & V & Vanadium & . & . & & 51,0 \\
\hline $\mathrm{Kr}$ & Krypton & . & . & $\cdot$ & 82.92 & W & Wolfram & . & . & & 184,0 \\
\hline La & Lanthan & . & . & 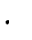 & 139,0 & $\mathrm{X}$ & Xenon & . & . & & 130,2 \\
\hline $\mathrm{Li}$ & Lithium & $\cdot$ & . & $\cdot$ & 6,94 & $\mathrm{Y}$ & Yttrium & . & & & 89,0 \\
\hline $\mathrm{Lu}$ & Lutetium & 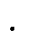 & . & 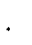 & 174,0 & $\mathrm{Yb}$ & Ytterbium & - & . & & 172.0 \\
\hline $\mathrm{Mg}$ & Magnesium & & . & $\cdot$ & 24,32 & $\mathrm{Zn}$ & Zink & . & ' & & 65,37 \\
\hline $\mathrm{Mn}$ & Mangan & 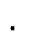 & . & $\cdot$ & 54,93 & $Z r$ & Zirkonium & . & . & & 90,6 \\
\hline Mo & Molybdän & . & 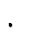 & 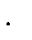 & 96,0 & & & & & & 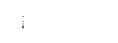 \\
\hline
\end{tabular}

